\section{Stimulus position and functional direction: Confounds in the concept of "bigger" in 5- and 6-year-olds}

\author{
GARY PHYE, Iowa State University, Ames, Iowa 50010 \\ and \\ TERRY TENBRINK, University of Missouri, Columbia, Mo. 65202
}

The use of the vertical linear dimension as a cue in paired comparison judgments of "bigger" with 5- and 6-year-olds was investigated, using drawings of real-world objects. The drawings were of equal area, and the objects depicted were ones to which a functional direction could be attributed. The results indjcated that the verfical dimension is a salient cue in the judgment of bigger. Also, the functional direction of an object appears to influence the selection of the vertical dimension as a cue. The difference in responding to displays having either a common or disparate baseline suggests that the inability to conserve length may be a factor influencing the judgment of "bigger" when stimulus objects do not share a common baseline.

In recent investigations of concept development, attention has been given to the development of the concept of "bigger" in 5-and 6-year-old children. Lumsden \& Poteat (1968) and Poteat $\&$ Hulsebus (1968) found the vertical dimension to be a predominate misleading perceptual cue when 5 - and 6-year-olds were asked to judge the bigger of two stimuli presented simultaneously. In both studies, when the areas for the stimulus pair were equal, the member with the greater vertical dimension was chosen as "bigger." This preference was exhibited when differences in the vertical dimension varied from 1 to $5^{1 / 2}$ in. across stimulus pairs. Hulsebus (1969), using a comparable procedure, noted a decrease in the influence of the vertical dimension with increasing age. This was in keeping with the performance of the college-age control group in the Poteat \& Hulsebus (1968) study. The college-age students were not influenced significantly by the vertical dimension. The stimuli in the aforementioned studies were either three-dimensional geometric objects or primarily planimetric geometric forms.

These studies have been interpreted as indicating that the preschool child responds to the word bigger primarily in terms of the vertical dimension. "This would suggest that the evolution of the child's concept of bigger is from a relatively simple one heavily weighting the vertical dimension to a multidimensional one approximating the adults concept of areal expanse or inferred volume [Lumsden \& Poteat, 1968 ]." It is the focusing on a single attribute or dimension in the process of making a judgment that eliminates the possibility of conservation in a Piaget-type conservation task. For example, the use of stimulus pairs with equal areas should not be confusing to 5- and 6-year-olds when they are asked "Which is bigger?" since the conservation of area, which is a multidimensional process, is typically beyond their cognitive ability.

Children of 5 and 6 are, according to the Piagetian frame of reference, at the intuitive stage of development. At this stage, the child's perceptions influence his judgments. It seemed plausible that, for certain objects commonly encountered in the environment, the functional movement or direction of an object (i.e., car, boat, or tree) could influence the selection of the linear dimension that would be selected as a cue in the judgment of "bigger."

The purpose of the present study was to investigate the salience of the vertical dimension in the paired comparison judgment of "bigger" with drawings of real-world objects. The drawings in each pair were of equal area. The objects selected as stimuli were ones to which a functional direction may be attributed. If the attributed functional direction of an object influenced the selection of the linear dimension used as a cue for the judgment of bigger, it would indicate an additional confounding source in the development of conservation of size for children of this age. \section{SUBJECTS}

Twenty-four 5- and 6-year-old Ss (range $=5$ years 4 months to 6 years 1 month; $\bar{X}=5$ years 8 months) were selected from the kindergarten program in the Child Development Department, Jowa State University.

\section{PROCEDURE}

A total of 12 stimulus displays were employed. Each stimulus display consisted of two line drawings on a 14 x 22 in. sheet of white posterboard. The areas for the two drawings in each pair were equal, but the vertical dimension was greater for one member and the horizontal dimension greater for the other. Since previous studies (Lumsden \& Poteat, 1968; Poteat \& Hulsebus, 1968; Hulsebus, 1969) had shown the disproportionate relevance of the vertical dimension when the difference in the vertical dimensions within a pair varied from 1 to 5.5 in., no attempt was made to control for differences across displays.

In the first 4 of the 12 displays, the functional direction of the represented objects was predominantly vertical; in the second group of 4 displays, the object's functional direction was predominantly horizontal. For the remaining 4 displays, neither the horizontal nor the vertical functional direction was judged by the Es to be dominant and was referred to as the ambiguous category.

The 12 displays and their dimensions are given in Table 1. For the objects in the ambiguous category, one view of the stocking cap was a top view and the other a side view. For the squirrel, one representation presented a front view of the animal seated on its hind legs and the other presented a view of the animal on all four feet. For the banjo and the rocket, one representation was vertical, the second was rotated $90 \mathrm{deg}$ to produce a

Table 1

Objects and Dimensions for Stimulus Displays

\begin{tabular}{rlll}
\hline Displays & \multicolumn{1}{c}{ Objects } & $\begin{array}{c}\text { Vertical by Horizontal Extent } \\
\text { Dimensions in Inches }\end{array}$ & Category \\
\hline 1 & Tree & $12 \times 4 ; 8 \times 6$ & Vertical \\
2 & Building & $11.5 \times 5 ; 5 \times 11.5$ & Vertical \\
3 & Men & $10.5 \times 4 ; 8.4 \times 5$ & Vertical \\
4 & Mug & $8 \times 5 ; 6.5 \times 6.2$ & Vertical \\
5 & Fish & $4.5 \times 5.5 ; 2.75 \times 7.9$ & Horizontal \\
6 & Turtle & $4.75 \times 7.9 ; 3.5 \times 10.7$ & Horizontal \\
7 & Boat & $5.5 \times 7.25 ; 3.75 \times 10.6$ & Horizontal \\
8 & Car & $5.75 \times 7 ; 3.75 \times 10.8$ & Horizontal \\
9 & Stocking Cap & $6.5 \times 6.5 ; 6.5 \times 6.5$ & Ambiguous \\
10 & Banjo & $12.0 \times 5.25 ; 5.25 \times 12.0$ & Ambiguous \\
11 & Squirrel & $7.75 \times 5 ; 5 \times 7.75$ & Ambiguous \\
12 & Rocket & $11 \times 5 ; 5 \times 11$ & Ambiguous \\
\hline
\end{tabular}


Table 2

Percentage of Ss Selecting as "Bigger" the Drawing Having the Greater Vertical Extent

\begin{tabular}{|c|c|c|c|c|c|c|c|}
\hline \multirow{2}{*}{\multicolumn{2}{|c|}{ Display }} & \multicolumn{2}{|c|}{ Set A } & \multicolumn{2}{|c|}{ Set B } & & \\
\hline & & \multicolumn{2}{|c|}{$\begin{array}{c}\text { Disparate Baseline } \\
(N=12)\end{array}$} & \multicolumn{2}{|c|}{$\begin{array}{l}\text { Common Borderline } \\
\qquad(N=12)\end{array}$} & \multicolumn{2}{|c|}{$\begin{array}{c}\text { Combined } \\
(N=24)\end{array}$} \\
\hline 1 & Tree & 100 & $(12)^{*}$ & 100 & $(12)^{*}$ & 100 & $(24)^{*}$ \\
\hline 2 & Building & 92 & $(11)^{*}$ & 100 & $(12)^{*}$ & 96 & $(23)^{*}$ \\
\hline 3 & Men & 92 & $(11)^{*}$ & 100 & $(12)^{*}$ & 96 & $(23)^{*}$ \\
\hline 4 & Mug & 100 & $(12)^{*}$ & 100 & $(12)^{*}$ & 100 & $(24)^{*}$ \\
\hline 5 & Fish & 8 & (1) & 42 & (5) & 25 & (6) \\
\hline 6 & Turtle & 17 & (2) & 50 & (6) & 33 & (8) \\
\hline 7 & Boat & 8 & (1) & 50 & (6) & 29 & (7) \\
\hline 8 & Car & 17 & (2) & 50 & (6) & 33 & (8) \\
\hline 9 & Cap & 67 & (8) & 83 & $(10)^{*}$ & 75 & $(18)^{*}$ \\
\hline 10 & Banjo & 75 & (9) & 92 & $(11)^{*}$ & 83 & $(20)^{*}$ \\
\hline 11 & Squirrel & 67 & (8) & 92 & $(11)^{*}$ & 79 & $(19)^{*}$ \\
\hline 12 & Rocket & 75 & (9) & 100 & $(12)^{*}$ & 88 & $(21)^{*}$ \\
\hline
\end{tabular}

horizontal perspective. The rationale for including the rocket was that children of this age have viewed rocket launchings on TV. The TV coverage includes not only the vertical ascent, but also the rocket's moving from left to right on the screen as it reaches the apex of its vertical ascent prior to earth orbit.

Two sets of stimulus displays were used, the drawings being identical in both sets. For Set $A$, the drawings were positioned so that there was not a common baseline for the two representations in each display. For Set $B$, the drawings shared a common horizontal baseline in each display. To avoid position effects, the drawing with the longe: vertical extent was on top for half of the displays in each category and on the bottom for the other half for Set A displays. For Set B displays, the figure with the longer vertical extent was on the right for half of the displays in each category and on the left for the remaining displays. The order in which the stimuli were presented was predetermined by counterbalancing within and between the categories. Half of the Ss were tested using Set A and half using Set B displays.

Experimental sessions were conducted in a room free from distractions, and Ss were tested individually. Ss were seated at a table $2 \mathrm{ft}$ from the display. As with previous studies, as each display was presented, the question was asked, "Which is bigger?" This form of the question allowed for the possible response, "They are the same." In no case did a $S$ respond in this manner. Each display was shielded from the S's view prior to introduction and was removed following a response in order to avoid comparisons between displays.

\section{RESULTS}

Responses to each display were evaluated using the chi-square statistic. As with previous studies, a 50:50 split was assumed for choices to each display. Table 2 presents results concerning the percentage of $S s$ choosing the drawing of greater vertical extent as "bigger" in each display. horizontal category of stimulus displays. In both Sets $A$ and $B$, the vertical extent did not exceed chance expectations. The stimulus displays in the ambiguous category for Set $\mathrm{A}$ also failed to exceed chance expectations.

Because the results did not indicate responses consistently favoring the drawing with the longer vertical extent, an analysis of variance was applied to the data in order to test for differences among conditions. The data were pooled across categories for each $S$, and a 2 (stimulus set) by 3 (category) analysis of variance with repeated measures was applied to the arcsin transforms of the vertical choice data. Arcsin transformations were used in order to increase the homogeneity of variance between cells. The result was a significant main effect of stimulus set $[F(1,22)=6.82, p<.01]$. Stimulus Set B, which consisted of displays with common baselines, received more responses to the drawings with the greater vertical extent than did Set A displays with disparate baselines. The main effect of category was also significant $[F(2,44)$ $=45.86, \mathrm{p}<.01]$. The individual category means were tested using the Newman-Keuls statistic. The means for the vertical, horizontal, and ambiguous categories were $2.79, .91$, and 2.27 , respectively. All means differed significantly from one another $(p<.05)$. The A by B interaction did not reach significance at the .05 level $[F(2,44)=2.57, p<.10]$.

The results of the chi-square supported to a limited degree the previous findings concerning the salience of the vertical linear
Of particular interest is the choice of objects with the longer

\section{DISCUSSION} analysis for the combined data dimension as a misleading cue in the judgment of bigger. The vertical linear dimension served as a salient cue in the vertical category for Set $A$ and Set $B$ displays and in the ambiguous category for Set B displays with a common baseline. It appeared that when there was congruence between the vertical functional direction of an object and the greater vertical extent, the vertical linear dimension was a predominant cue. When the directional function of an object was ambiguous, the vertical extent served as a salient cue only when the stimulus displays had a common horizontal baseline.

The responses to the horizontal category are of interest in that the choices for the drawing with the greater vertical extent did not exceed chance expectations. When there was a horizontal directional function, the vertical extent did not serve as a salient cue, the implication here being that the functional direction of a common environmental object influenced the choice of the linear dimension that would serve as a cue for the judgment of "bigger."

The significant main effect of stimulus display in the analysis of variance indicated a difference in responding to the display sets. Choices favoring the vertical extent were significantly greater for Set B displays having a common baseline. This raises a question concerning the influence of a common or disparate baseline in size judgments with children at this stage of cognitive development. It would appear that the placement of objects in a paired comparison situation is confounded with the selection of the vertical linear extent. The effect of this confounding is a decrease in the influence of the vertical linear dimension as a cue. The influencing factor here could possibly be an inability to conserve length. A nonconserver of length exhibits an inability to focus on both ends of an object when it is contrasted with a standard or another object in a paired comparison situation. By focusing only on the discrepancy in length at one extreme, he fails to consider the discrepancy at the other extreme and compensate accordingly. With displays that have a common baseline, conservation of length would not be a consideration. With Set A displays, conservation of length would be a necessary operation and, for nonconservers, a confound in the judgment of "bigger."

The significance of the main effect of category is viewed as supporting the contention that the functional direction of an object influences the selection of the vertical linear dimension. The choices for the drawings with the longer vertical 
extent were greatest for the vertical category with objects having a vertical functional direction. The ambiguous category where the functional direction was ambiguous received the second largest number of choices for the drawings with the longer vertical extent, and the horizontal category where the horizontal functional direction predominated received the fewest choices. The vertical linear dimension as a salient cue is confounded with the functional direction of an object. When the functional direction of an object is predominantly horizontal, the salience of the vertical linear dimension as a cue is diminished.

In conclusion, for 5- and 6-year-olds the vertical linear dimension was a salient misleading cue in the judgment of "bigger" of common objects. Additionally, the use of a disparate baseline and the functional direction of an object appeared to be confounded with the selection of the vertical linear dimension. The effect in both cases was the diminution of the jnfluence of the vertical linear dimension as a salient cue. The hypothesis was tendered that the inability to conserve length may be a source of confounding in a paired comparison choice of "bigger" when the stimulus pair does not share a common baseline.

\section{REFERENCES}

HULSEBUS, $R, C$. An interaction between vertical dimension and age in children's judgments of size. Perceptual \& Motor Skills, $1969,28,841-842$.

LUMSDEN, E. A.. \& POTEAT, B. W. S. The salience of the vertical dimension in the concept of bigger in five- and six-year olds. Journal of Verbal Learning \& Verbal Behavior, 1968. 7, 404-408.

POTEAT, B. W. S.. \& HULSEBUS, R. C. The vertical dimension: A significant cue in the preschool child's concept of bigger. Psychonomic Science, 1968, 12, 369-370. 